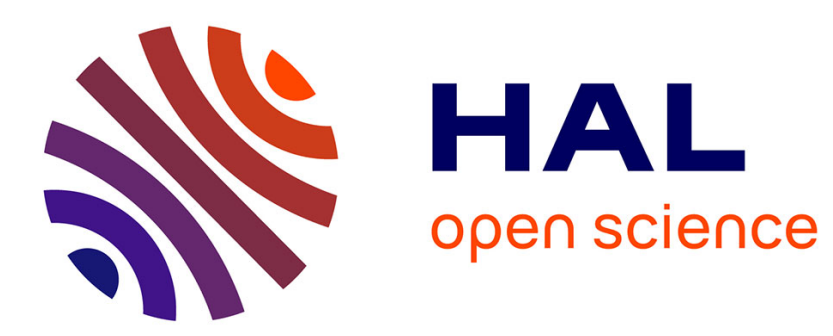

\title{
Comment tirer tous les bénéfices de la collaboration industrie-recherche?
}

\author{
Nathalie Fabbe-Costes
}

\section{To cite this version:}

Nathalie Fabbe-Costes. Comment tirer tous les bénéfices de la collaboration industrie-recherche?.

Supply Chain Magazine, 2016, Nº110, Décembre 2012, pp.96-97. hal-01424029

\section{HAL Id: hal-01424029 \\ https://hal-amu.archives-ouvertes.fr/hal-01424029}

Submitted on 1 Jan 2017

HAL is a multi-disciplinary open access archive for the deposit and dissemination of scientific research documents, whether they are published or not. The documents may come from teaching and research institutions in France or abroad, or from public or private research centers.
L'archive ouverte pluridisciplinaire HAL, est destinée au dépôt et à la diffusion de documents scientifiques de niveau recherche, publiés ou non, émanant des établissements d'enseignement et de recherche français ou étrangers, des laboratoires publics ou privés. 


\section{Comment tirer tous les bénéfices de la collaboration industrie-recherche?}

De nombreuses entreprises ou organisations s'engagent dans des collaborations avec des chercheurs, y compris avec des projets touchant à des aspects organisationnels ou stratégiques. De leur côté, les chercheurs sont invités à plus collaborer avec des acteurs publics ou privés. Si les mérites de ce type de collaboration sont multiples, elle ne va pas de soi et ne s'improvise pas. Nous dégageons de nos expériences de collaborations industrie-recherche menées au Cret-Log dans le domaine de la logistique et du Supply Chain Management (SCM) quelques principes pour en tirer tous les bénéfices.

L a recherche en logistique et SCM a une longue tradition de travail en collaboration avec les entreprises et les institutions. Comme en témoignent les billets du Cret-Log, la plupart des recherches en logistique et SCM ont des impacts managériaux, et il n'est pas une thèse qui ne développe ses apports pour les entreprises et plus largement pour la société. Ce qui semble " naturel " dans notre domaine, tend à devenir une règle dans la mesure où la plupart des appels à projets de recherche, que ce soit au niveau des Régions, des pôles de compétitivité, de l'Agence Nationale de la Recherche (ANR) ou de l'Europe (programme H2020), encouragent, voire imposent, la collaboration avec des entreprises ou parties prenantes socio-économiques. La frontière entre recherche fondamentale et appliquée tend à s'estomper.

Mais, si la collaboration industrie-recherche se développe (le terme industrie renvoyant aux entreprises et organisations au sens large), elle n'est pas toujours gage de succès ni source de satisfactions pour les partenaires. Pour qu'un projet soit réussi, il faut en effet qu'il crée de la valeur pour les entreprises et la société, et qu'il fasse progresser la connaissance scientifique. Or il n'est pas si facile d'atteindre conjointement ces 2 types de bénéfices, notamment car les logiques de travail, les résultats attendus et les motivations des partenaires sont parfois difficiles à concilier. Réussir un projet qui associe industrie et recherche demande de nombreuses interac-

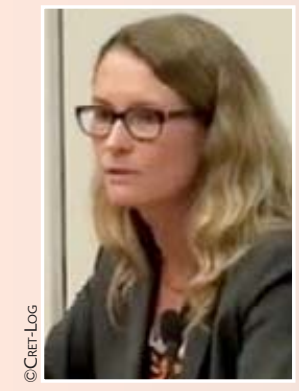

Nathalie Fabbe-Costes

Professeur des Universités,

Directrice du Cret-Log,

Aix-Marseille Université (AMU)

nathalie.fabbe-costes@univ-amu.fr tions tout au long du projet qui sont, de notre point de vue, la clé du succès.

\section{L'initialisation du projet pour clarifier les objectifs}

Il est indispensable, avant de s'engager, de clarifier les objectifs recherchés par les parties prenantes, ce qui permet de vérifier leur compatibilité. Le caractère stratégique d'un projet pour l'ensemble des parties est souvent un gage d'engagement dans sa réalisation. La préparation du projet permet aussi de définir un design de recherche qui concilie les objectifs des parties, sachant qu'il ne s'agit pas d'avoir les mêmes objectifs, mais que ceux-ci soient compatibles. Travailler le design de la recherche conduit à réfléchir à la manière de s'impliquer et d'interagir tout au long du projet. Il conduit aussi à rendre compatibles les horizons de travail (les questions de délais pour produire les résultats attendus étant un point souvent problématique) et à définir les informations qui seront partagées. Au-delà de ces aspects souvent pratiques de la gestion des projets de recherche, il n'est pas inutile de s'assurer que les partenaires parlent des mêmes choses et convergent sur l'exécution à réaliser. Comme dans le Petit Prince, praticiens et académiques ont souvent besoin d'un peu de temps pour s'apprivoiser, ce qui passe par une discussion sur les concepts clés de la recherche, les partis pris méthodologiques et hypothèses fondamentales, leurs visions du monde, etc.

\section{La contractualisation,}

\section{un point clef à ne pas négliger}

Au cœur du dispositif, le contrat ou la convention de recherche joue un rôle déterminant auquel les chercheurs et les entreprises n'accordent pas toujours l'importance qu'il faudrait. Rappelons qu'il existe de nombreuses manières d'organiser une collaboration industrie-recherche : contrats de recherche en lien avec des appels à projets (ex : PreditT, ANR, H2020), contrats de prestation avec des filiales de valorisation (ex : Protisvalor à Aix-Marseille Université), 
chaires dans le cadre de fondations (ex : celle de SNCF Logistics avec AMU), embauche de doctorants sur la base de contrats privés ou de conventions Cifre (avec l'ANRT). Souvent vu comme une étape strictement règlementaire, fastidieuse et contraignante, source de retard au démarrage des projets, il est en fait l'occasion pour les partenaires de s'interroger sur les enjeux de la recherche et les résultats attendus, d'échanger sur leur valorisation, de choisir les modalités de la collaboration, d'ajuster les méthodes de travail et la manière de conduire la recherche, les moyens à lui allouer et la manière de résoudre les éventuels problèmes, notamment en lien avec la confidentialité ou la propriété intellectuelle... des clarifications qui ne sont pas inutiles !

\section{La réalisation de la recherche et la production des résultats}

Un projet de recherche comporte toujours une part de risque et d'incertitude, et nécessite souvent d'être réorienté en cours de route. Le pilotage des projets de grande envergure compte de nombreux jalons qui s'accompagnent de décisions aux conséquences généralement irréversibles. Une traçabilité du déroulement du projet ainsi que du dialogue entre les partenaires sont indispensables pour prendre des décisions qui conviennent aux parties prenantes et redéfınir ensemble, si nécessaire, l'orientation du projet. La production des résultats est aussi un moment important où les interactions sont source d'avancées, certains résultats ne pouvant être produits que dans l'interaction. L'analyse conjointe des résultats obtenus, qui révèlent souvent des surprises, est aussi importante pour la fabrication, le partage et l'appropriation des connaissances en lien avec le projet. Finalement, l'analyse ex post des résultats et des motifs de satisfaction / insatisfaction est un temps fort des projets à ne pas négliger pour tirer parti de l'expérience et peut-être la renouveler !

\section{Identifier les questions de demain}

S'engager dans une collaboration industrie-recherche est une aventure passionnante. Les interactions y sont source de richesse pour les parties prenantes. Elles sont aussi le gage du succès. Loin d'être une perte de temps, le dialogue et la co-construction des projets, pour en poser de solides bases et les

\section{Côté industrie}

Développement d'activités

Aide à la décision

Contribution à la R\&D

Innovation

Absorption de connaissances

Veille et prospective

Stimulation type « think tank »

Côté recherche

Travailler sur des " cas "

Bénéficier de données "réelles "

Acquérir des connaissances de terrain Développer des idées, des modèles Stabiliser des concepts Développer/tester des théories Identifier de nouveaux sujets de recherche

\section{Tableau 2. Un dialogue et une co-construction à chaque étape du projet}

Le dialogue porte sur :

Les objectifs du projet et résultats attendus La problématique, les concepts clés La(les) méthodologies possibles Les théories, l'épistémologie

La formule de collaboration Le processus de recherche Les rôles et responsabilités Planning détaillé de la recherche Livrables de la recherche et délais

La production et le partage des données et connaissances La traçabilité du processus Les problèmes rencontrés

Les retards, les blocages,

leur cause

Les réorientations éventuelles

Les aspects stratégiques liés aux résultats du projet L'analyse des " surprises »

La satisfaction des partenaires Les projets futurs
La co-construction

Définir ensemble les orientations de la recherche

S'assurer que les partenaires se comprennent Aligner le projet avec les objectifs Anticiper les difficultés potentielles

Co-concevoir un projet faisable et valable pour les partenaires

Co-production de la recherche (selon les interactions prévues)

Co-piloter la recherche (jalons) Prendre ensemble les décisions de réorientation

et analyser leur impact

Coproduire les résultats et analyses

Discuter de la pertinence/valeur des résultats

Faire ensemble un bilan

Alimenter l'apprentissage/

l'absorption (s'apprivoiser)

ajuster chemin faisant, permettent de tirer les bénéfices de la collaboration et de produire les résultats attendus. La collaboration industrie-recherche nous semble d'autant plus importante en logistique que les Supply Chains, par nature inter-organisationnelles, gagnent à être étudiées par des acteurs " neutres ", que si les chercheurs aident à résoudre les problèmes posés par les entreprises, ils peuvent aussi les aider à poser différemment certains problèmes, notamment dans le domaine du management. Enfin, s'ils savent traiter des questions logistiques bien identifiées, ils peuvent identifier les questions de demain (en lien avec l'économie collaborative ou circulaire, l'internet des objets, les technologies de demain, les ruptures sociétales ou politiques, etc.). Alors qu'attendez-vous pour collaborer avec la recherche?

\section{Pour en savoir plus.}

The power of co-construction and dialogue to interact with industry in strategic logistics, OM and SCM research projects, N. Fabbe-Cstes, communication at the $23^{\text {rd }}$ International Annual EuroMA Conference "Interactions ", $17^{\text {th }}-22^{\text {nd }}$ June 2016. hosted by the Norwegian University of Science and Technology (NTNU), Trondheim, Norway. 